\title{
In vitro pharmacodynamics of a danofloxacin plus colistin combination against multidrug-resistant (MDR) Escherichia coli isolated from animals
}

\author{
๑ Murat Cengiz ${ }^{1, *}, \oplus$ Pinar Sahinturk ${ }^{1,2}, \oplus$ Gulce Hepbostanci $^{2}$, \\ $\odot$ Halis Akalin ${ }^{3}$, $\odot$ Songul Sonal ${ }^{a}$
}

aLaboratory of Molecular Pharmacology, Department of Pharmacology and Toxicology, Faculty of Veterinary Medicine, Uludag

University, 16059, Nilufer, Bursa, Turkey, bInstitute of Health Science, Uludag University, 16059, Nilufer, Bursa, Turkey

cDepartment of Infectious Diseases and Clinical Microbiology, Faculty of Medicine, Uludag University, 16059, Nilufer, Bursa,

Turkey

Received 04.07.2019 Accepted 23.09.2019

\begin{abstract}
As an alternative antimicrobial combination, danofloxacin+colistin was used for the inhibition of multidrug resistant E.coli. After evaluation of interaction between the drugs by fractional inhibitory concentration tests and time kill assays, antimicrobial activity of the combination was showed by in vitro pharmacodynamics tests (minimum bactericidal concentration and mutant prevention concentration). Post-antibiotic and post-antibiotic sub-MIC effects were also determined in this study. In synergism tests, danofloxacin+colistin was found highly synergistic (\%87) against E.coli isolates from animal origin. The combination exerted bactericidal activity against all E.coli isolates and individual bactericidal activity of each compound was lower than the combination. The combination reduced mutant prevention concentration of danofloxacin and colistin up to 32 -fold. Post-antibiotic sub-MIC effects of the combination at all sub-MIC concentrations were significantly longer than the post-antibiotic effects of combination $(\mathrm{p}<0.001)$, danofloxacin $(\mathrm{p}<0.001)$ and colistin $(\mathrm{p}<0.001)$. The results of this study showed that danofloxacin+colistin combination can be reserved as an alternative drug combination against MDR E.coli in veterinary medicine.
\end{abstract}

Keywords: Colistin, Danofloxacin, in vitro Pharmacodynamics, MDR E.coli

\section{Introduction}

Multidrug resistant (MDR) Escherichia coli (E.coli) is important for public health due to high risk for the treatment of infectious diseases at available dosage regimens. ${ }^{1}$ To combat with MDR E.coli is one of the most challenging problems in infectious diseases. ${ }^{2}$ In cases involving a lack of effective agents, antimicrobial combinations can be used for the treatment of infectious diseases causing by resistant strains.2 For instance, polymyxins induce rapid changes in the permeability of the cytoplasmic membrane of Gram-negative bacilli, thereby other antimicrobial agents can enter into the cell. ${ }^{3,4}$ Therefore, colistin (CST) can be used in combination with other antibiotics to achieve a synergistic effect. ${ }^{5}$ In veterinary medicine, CST is individually used for the treatment of infections caused by Enterobacteriaceae in various animals. The use of CST in animals can select for CST resistant Enterobacteriaceae which have the potential to be transmitted to humans. Therefore, CST sales for use in animals should be reduced. ${ }^{6}$ Decades of CST use in veterinary medicine have not been associated with increased resistance prevalence in E.coli isolated from animals. ${ }^{7}$ However, the plasmid-mediated CST resistance gene (mcr-1) creates a new threat due to the transferability of CST resistance between bacterial strains and species. ${ }^{8}$ Fluoroquinolones (FQs) are synthetic anti-

* Corresponding author: Murat Cebgiz, Laboratory of Molecular Pharmacology, Department of Pharmacology and Toxicology,

Faculty of Veterinary Medicine, Uludag University, 16059, Nilufer, Bursa, Turkey

Phone number: (+90) 2242941323 E-mail address: cengizm@uludag.edu.tr 
microbials that exhibit a concentration-dependent bactericidal effect by inhibiting bacterial topoisomerase enzymes. Danofloxacin (DAN) is a synthetic second-generation FQ with broad-spectrum antibacterial activity and is used in the treatment of respiratory disease in few animal species. ${ }^{9}$

There are some reported drug interactions between FQs and CST that have significance in human medicine. For instance, the CST-ciprofloxacin (CIP) combination therapy was found efficient against MDR P. aeruginosa and K. pneumoniae strains. ${ }^{5,10}$ A synergistic or indifferent effect between CST and levofloxacin (LVX) was also observed in vitro and in vivo against CST-susceptible A. baumannii strains. ${ }^{11}$

Based on the EMA/CVMP/261180/2012 Guideline, the data required to demonstrate the therapeutic efficacy of an antimicrobial agent include minimum inhibitory concentration (MIC), minimum bactericidal concentration (MBC) and kinetics of bacterial killing, fractional inhibitory concentration (FIC) and other pharmacodynamic variables such as post-antibiotic effect (PAE) and post-antibiotic sub-minimum inhibitory concentration effect (PASME). Mutant prevention concentration (MPC) testing provides practical information on the drug concentrations necessary to restrict mutant growth. ${ }^{12}$ The PAE is defined as the length of time that bacterial growth is suppressed following limited exposure to an antibiotic. ${ }^{13}$ PA-SME is defined as the time interval that includes the PAE plus the additional time during which growth is suppressed by sub-MICs. The objective of this work was to investigate possible interaction between DAN and CST against MDR E.coli, and to collect pharmacological data in order to show antimicrobial activity of the potential combination by performing in vitro pharmacodynamics tests.

\section{Material and methods}

\section{Bacterial strains}

For this study, six representative isolates with animal origin were chosen from different patterns based on resistance profile and genotype. The susceptibility profiles of the E.co$l i$ isolates were given in Table 1. Five of the six E.coli isolates had MDR profile. E.coli ATCC25922 and E.coli AG100 were used as control strain.

\section{Fractional inhibitory concentration index (FICI)}

The FICIs of the DAN and CST combinations were determined using the checkerboard method. ${ }^{14}$ Dilutions ranging from $1 / 32 x M I C$ to $4 x M I C$ were tested for each antimicrobial. The FICI was interpreted as follows: FICI $\leq 0.5=$ synergy; FICI $>4.0=$ antagonism; FICI $\quad 0.5-4=$ indifference $/$ additive. FIC index/indices were calculated as follows: FICA = MIC drug A in combination / MIC drug A alone $\mathrm{FICB}=\mathrm{MIC}$ drug $\mathrm{B}$ in combination / MIC drug $\mathrm{B}$ alone FIC index $/ \Sigma$ FIC $=$ FICA + FICB

\section{Time-kill experiments}

Time-kill experiments were performed as described previously. ${ }^{15}$ Synergy was defined as a $\geq 2 \log 10$ decrease in the colony count at 6 or $24 \mathrm{~h}$ with the combination treatment compared with the initial inoculum. The drug combination was considered to be antagonistic if there was a $\geq 2$ $\log _{10}$ increase in $\mathrm{cfu} / \mathrm{ml}$, and a $<2 \log _{10}$ change in $\mathrm{cfu} / \mathrm{ml}$ was interpreted as indifference.

\section{Minimum bactericidal concentration (MBC)}

The MBCs of the antimicrobials and the combination were determined using broth microdilution method as described previously by Hansen and Bloundeau. ${ }^{16}$ Overnight cultures of E.coli isolates were inoculated in Mueller-Hinton Broth (MHB) containing antimicrobials and combination in the range of 1-16 x MIC/FIC, and incubated at 37 ${ }^{\circ} \mathrm{C}$ for $16-20 \mathrm{~h}$. The MBC was the lowest concentration that inhibits $99.9 \%$ of the culture and determined by plating $100 \mu \mathrm{l}$ of sample onto Tryptic Soy Agar (TSA).

\section{Mutant prevention concentration (MPC)}

The MPCs of the antimicrobials and the combination were determined as described previously by Blondeau et al. ${ }^{16}$ Briefly, each E.coli isolate and control strain was freshly grown from stock stored at $-25^{\circ} \mathrm{C}$. Strains were incubated overnight at $37^{\circ} \mathrm{C}$ in $100 \mathrm{ml}$ of $\mathrm{MHB}$, after which the cultures were centrifuged at $9000 \mathrm{rpm}$ for 15 minutes. The supernatant was discarded and pellet was resuspended in 3 $\mathrm{ml}$ of MHB to achieve $>10^{10} \mathrm{cfu} / \mathrm{ml}$. A $100 \mu \mathrm{l}$ aliquot of this culture was used to inoculate plate count agar plates containing serial dilutions of the antimicrobials or the combination. The plates were incubated at $37^{\circ} \mathrm{C}$ for $72 \mathrm{~h}$ and examined every $24 \mathrm{~h}$ for growth of E.coli. The MPC was determined as the concentration that allowed no growth of bacteria at the end of the 72-h incubation. Each experiment was conducted in duplicate.

The mutant selection window (MSW) was determined as the concentration difference of drug between MIC and MPC. The mutant prevention index (MPI) was defined as MPC:MIC ratio.

\section{Post-antibiotic effect and post-antibiotic sub-MIC effect}

An inoculum of approximately $5 \times 10^{7} \mathrm{cfu} / \mathrm{ml}$ was exposed to each drug (alone or combination) at concentrations of $1 \mathrm{xMIC}$ and $4 \mathrm{xMIC}$ for $1 \mathrm{~h}$ at $37^{\circ} \mathrm{C}$ in a shaking incubator, 
followed by washout, centrifugation and resuspension in $10 \mathrm{ml}$ of $\mathrm{MHB}$ and incubation at $37^{\circ} \mathrm{C}$, as described previously. ${ }^{17}$ Growth was monitored hourly for $6 \mathrm{~h}$ by performing serial dilutions, and determining cfu of the sample per milliliter on Mueller-Hinton Agar (MHA). The PAE was measured by using the equation PAE (in hours) $=\mathrm{T}-\mathrm{C}$, where $\mathrm{T}$ is the time required for the treated organisms to grow $1 \log$ unit and $\mathrm{C}$ is the time needed for the organisms with no drug exposure to grow $1 \log$ unit, as described previously. ${ }^{13}$

In cultures designated for PA-SME, the PA-phase E.coli isolates were exposed to different sub-MICs $(0.1,0.2$ and $0.3 \mathrm{xMIC}$ ) of each drug (alone or combination). All samples were incubated in at $37^{\circ} \mathrm{C}$ in a shaking incubator and the growth of all cultures was monitored by determining cfu, as described above for PAE. The PA-SME was calculated by using the equation PA-SME (in hours) $=\mathrm{T}_{\mathrm{PA}}$ - C, where $\mathrm{T}_{\mathrm{PA}}$ is the time required for sub-MIC-treated PA-phase organisms to grow $1 \log$ unit and $\mathrm{C}$ is the time required for control organisms to grow 1 log unit, as described previously. ${ }^{13}$

\section{Statistical analysis}

The statistical analysis was conducted using SPSS Statistics 22. One-way ANOVA followed by LSD multiple comparisons test was performed to examine the change in PAE and PA-SME values of drug concentrations alone and in combination. $\mathrm{p}<0.05$ was considered statistically significant.

\section{Results}

The FIC values of DAN+CST combination for MDR E.coli isolates are shown in table 1 . The FICIs were found in the range of 0.15-2.03. The incidence of synergy and indifference was $83 \%$ and $17 \%$, respectively. Antagonism was not detected for any of E.coli isolates by the checkerboard method.

The results of time-kill assays are shown in table 1. At the $6 \mathrm{~h}$ incubation time point, the combination therapy resulted in a $\geq 3 \log 10$ reduction in viable counts against 5/6 MDR E.coli isolates, and bactericidal synergic activity was observed (figure 1). Antagonism was observed only for E.coli E222, which has two mutations in gyrA. At the 24 $\mathrm{h}$ incubation time point, DAN+CST combination exerted antagonism against 5/6 MDR E.coli isolates and synergistic activity was observed only for the most susceptible isolate, E.coli E175 (figure 1). Regrowth was observed at $24 \mathrm{~h}$ for 5/6 MDR E.coli isolates.

The MBC values are shown in table 2. The lowest MBC $(0.064 \mu \mathrm{g} / \mathrm{ml})$ was recorded for the most susceptible isolate, E.coli E175. The MBC values for the rest of the E.coli isolates were 4 or $8 \mu \mathrm{g} / \mathrm{ml}$. The MBCs of CST ranged from 2 to $128 \mu \mathrm{g} / \mathrm{ml}$. Based on the MBC:MIC ratios, antimicrobial effect was interpreted as bactericidal (MBC:MIC/FIC=14) or bacteriostatic (MBC:MIC/FIC $\geq 8$ ). The bactericidal effect rates of DAN, CST and DAN+CST were 100\%, 66\%

Figure 1. Time-kill curve of danofloxacin+colistin combination against MDR E.coli isolates and control strains

\section{DAN/CST}

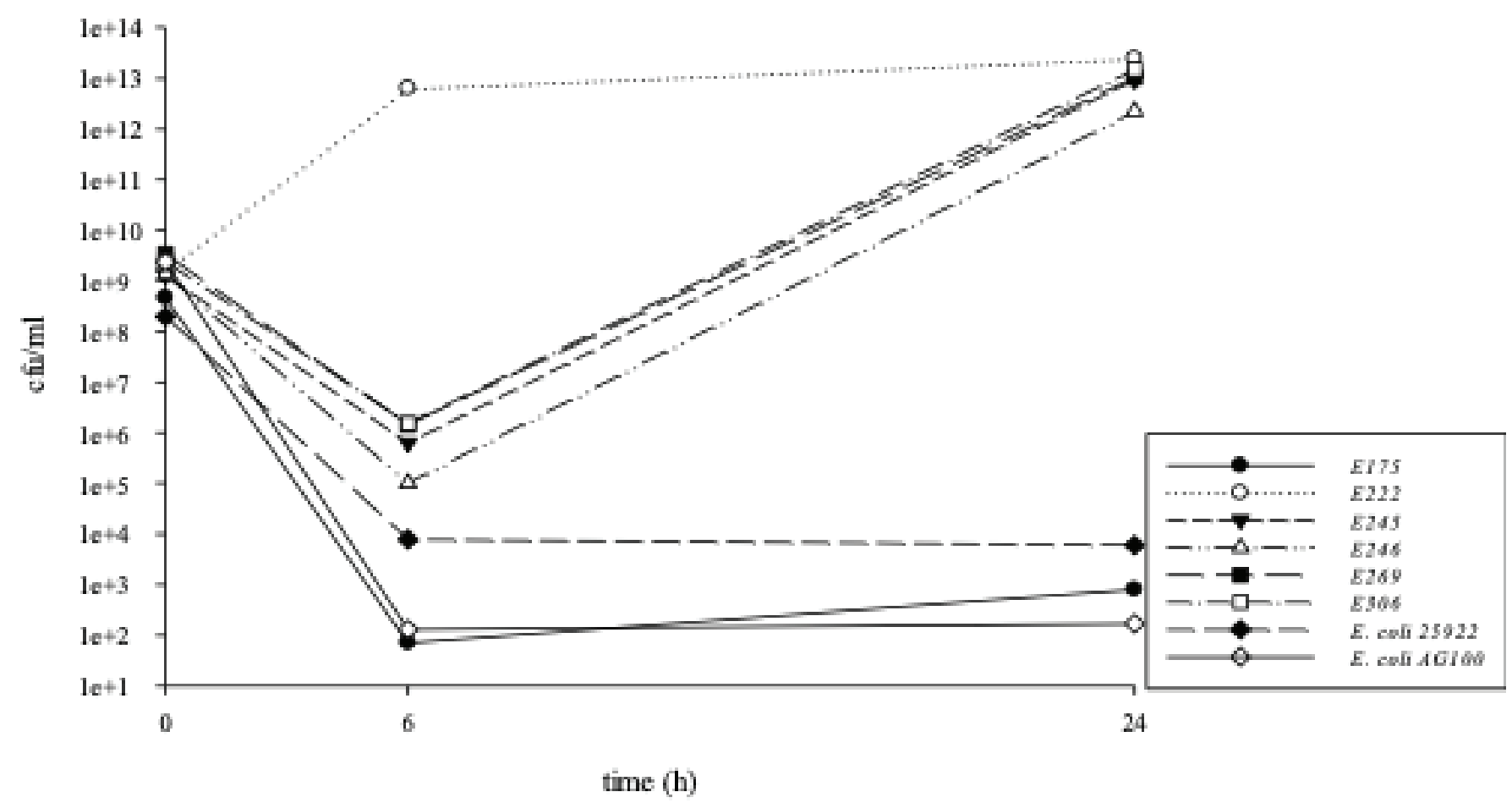


Table 1

\begin{tabular}{|c|c|c|c|c|c|c|c|c|c|c|c|c|c|c|c|}
\hline & & \multicolumn{7}{|c|}{ Resistance mechanism } & \multicolumn{3}{|c|}{ Checkerboard } & \multicolumn{4}{|c|}{ Time-kill } \\
\hline & & QRDR & & PMQR & MDR $^{\mathrm{a}}$ & & & & & & & $\log r$ & uction & Interp & tation \\
\hline $\begin{array}{l}\text { Isolate } \\
\text { ID }\end{array}$ & $\begin{array}{l}\text { Resistance } \\
\text { profile }\end{array}$ & gyrA & parC & $o q x B$ & $\operatorname{mar} A$ & $a c r B$ & soxS & ompF & $\mathrm{DAN} / \mathrm{CST}^{\mathrm{b}}$ & FICI & Interpretation & $6 \mathrm{~h}$ & $24 \mathrm{~h}$ & $6 \mathrm{~h}$ & $24 \mathrm{~h}$ \\
\hline E175 & SMX & & & & $\downarrow \downarrow$ & $\downarrow$ & $\downarrow \downarrow$ & $\uparrow$ & $0.002 / 4$ & 2.03 & ADD & 6.82 & 5.76 & SYN & SYN \\
\hline E222 & $\begin{array}{l}\text { NAL, CIP, } \\
\text { SMX, TMP, } \\
\text { TET, OTC, } \\
\text { CHL }\end{array}$ & Ser83Leu & Ser80Ile & & $\downarrow \downarrow$ & $\downarrow \downarrow$ & $\uparrow \uparrow$ & $\uparrow$ & $0.064 / 1$ & 0.50 & SYN & -3.59 & -4.18 & ANT & ANT \\
\hline E245 & $\begin{array}{l}\text { NAL, CIP, } \\
\text { ORB, GAT, } \\
\text { AMP, CEF, } \\
\text { GEN, TET, } \\
\text { OTC, ERY, } \\
\text { CHL }\end{array}$ & $\begin{array}{l}\text { Ser83Leu } \\
\text { Asp87Glu }\end{array}$ & & & $\uparrow$ & $\uparrow \uparrow$ & $\uparrow \uparrow$ & $\uparrow$ & $0.064 / 1$ & 0.50 & SYN & 3.32 & -3.86 & SYN & ANT \\
\hline E246 & $\begin{array}{l}\text { NAL, GAT, } \\
\text { AMP, TMP, } \\
\text { GEN, TET, } \\
\text { OTC, CHL, } \\
\text { CST }\end{array}$ & Ser83Leu & & & $\uparrow \uparrow$ & $\uparrow \uparrow$ & $\uparrow \uparrow \uparrow$ & $\downarrow \downarrow \downarrow$ & $0.032 / 1$ & 0.15 & SYN & 4.14 & -3.21 & SYN & ANT \\
\hline E269 & $\begin{array}{l}\text { NAL, SMX, } \\
\text { TMP, TET, } \\
\text { OTC, CST }\end{array}$ & & & & $\downarrow \downarrow$ & $\downarrow$ & $\uparrow \uparrow$ & $\uparrow$ & $0.064 / 1$ & 0.31 & SYN & 3.38 & -3.49 & SYN & ANT \\
\hline E306 & $\begin{array}{l}\text { NAL, CIP, } \\
\text { ORB, AMP, } \\
\text { TMP, TET, } \\
\text { OTC, ERY, } \\
\text { CHL }\end{array}$ & Ser83Thr & & + & $\downarrow \downarrow$ & $\downarrow \downarrow$ & $\uparrow$ & $\uparrow$ & $0.064 / 1$ & 0.50 & SYN & 3.21 & -3.79 & SYN & ANT \\
\hline
\end{tabular}

Table 2

\begin{tabular}{|c|c|c|c|c|c|c|c|c|c|c|c|c|c|c|c|c|c|c|}
\hline \multirow[t]{3}{*}{ Isolate ID } & \multicolumn{18}{|c|}{ Pharmacodynamic parameters } \\
\hline & \multicolumn{3}{|c|}{ MICs $(\mu \mathrm{g} / \mathrm{ml})$} & \multicolumn{3}{|c|}{ MBCs $(\mu \mathrm{g} / \mathrm{ml})$} & \multicolumn{3}{|c|}{ MBC:MIC } & \multicolumn{3}{|c|}{ MPCs $(\mu \mathrm{g} / \mathrm{ml})$} & \multicolumn{3}{|c|}{ MPIs } & \multicolumn{3}{|c|}{ MSWs $(\mu \mathrm{g} / \mathrm{ml})$} \\
\hline & DAN & CST & $\mathrm{DAN}+\mathrm{CST}$ & DAN & CST & $\mathrm{DAN}+\mathrm{CST}$ & DAN & CST & $\mathrm{DAN}+\mathrm{CST}$ & DAN & CST & $\mathrm{DAN}+\mathrm{CST}$ & DAN & CST & $\mathrm{DAN}+\mathrm{CST}$ & DAN & CST & $\mathrm{DAN}+\mathrm{CST}$ \\
\hline E175 & 0.064 & 2 & $0.002 / 4$ & 0.064 & 2 & $0.002 / 2$ & 1 & 1 & 1 & 0.512 & 32 & $0.016 / 32$ & 8 & 16 & 8 & $0.064-0.512$ & $2-32$ & $0.002-0.016 / 4-32$ \\
\hline E222 & 2 & 2 & $0.064 / 1$ & 8 & 2 & $0.064 / 1$ & 4 & 1 & 1 & 8 & 64 & $4 / 64$ & 4 & 32 & 64 & $2-8$ & $2-64$ & $0.064-4 / 1-64$ \\
\hline E245 & 2 & 2 & $0.064 / 1$ & 4 & 32 & $1 / 16$ & 4 & 16 & 16 & 32 & 256 & $4 / 64$ & 16 & 128 & 64 & $2-32$ & $2-256$ & $0.064-4 / 1-64$ \\
\hline E246 & 1 & 8 & $0.032 / 1$ & 4 & 128 & $0.512 / 16$ & 4 & 16 & 16 & 32 & 256 & $1 / 32$ & 32 & 32 & 32 & $1-32$ & $8-256$ & $0.032-1 / 1-32$ \\
\hline E269 & 1 & 4 & $0.064 / 1$ & 4 & 4 & $0.064 / 1$ & 4 & 1 & 1 & 16 & 32 & $1 / 16$ & 16 & 8 & 16 & $1-16$ & $4-32$ & $0.064-1 / 1-16$ \\
\hline E306 & 1 & 2 & $0.064 / 1$ & 4 & 2 & $0.064 / 1$ & 4 & 1 & 1 & 4 & 64 & $2 / 32$ & 4 & 32 & 32 & $1-4$ & $2-64$ & $0.064-2 / 1-32$ \\
\hline
\end{tabular}

Table 3

\begin{tabular}{|c|c|c|c|c|c|c|c|c|c|}
\hline \multirow[t]{2}{*}{ Isolates } & \multirow[t]{2}{*}{ Antibiotic } & \multicolumn{2}{|c|}{ PAEs (h) } & \multicolumn{3}{|c|}{ 1xPA-SMEs (h) } & \multicolumn{3}{|c|}{ 4xPA-SMEs (h) } \\
\hline & & $1 \mathrm{xMIC}$ & $4 \mathrm{xMIC}$ & 0.1 & 0.2 & 0.3 & 0.1 & 0.2 & 0.3 \\
\hline \multirow[t]{3}{*}{ E. coli 25922} & DAN & 5.9 & 6.6 & 4.5 & 0.8 & 3 & $>24$ & $>24$ & $>24$ \\
\hline & $\mathrm{CST}$ & 2.1 & 9.0 & 11.2 & 12.4 & 12.2 & 4.3 & 4.0 & 3.5 \\
\hline & $\mathrm{DAN}+\mathrm{CST}$ & 4.4 & - & 2.2 & 1.9 & 2.3 & - & - & - \\
\hline \multirow[t]{3}{*}{$E 175$} & DAN & 1.9 & 4.5 & 7.4 & 10.9 & 13.2 & 3.8 & 8.7 & 7.8 \\
\hline & CST & 0.4 & 0.9 & 1.2 & 1.1 & 1.3 & 1.1 & 1.2 & -0.1 \\
\hline & $\mathrm{DAN}+\mathrm{CST}$ & 2.5 & - & 1.6 & 1.7 & 1.7 & - & - & - \\
\hline \multirow[t]{3}{*}{ E222 } & DAN & 1.7 & 1.8 & 0.3 & 0.3 & 0.8 & 1.3 & 1.3 & 2.6 \\
\hline & $\mathrm{CST}$ & 1.2 & 1.0 & 4 & 4.5 & 5.2 & 1.2 & 1.6 & 1.7 \\
\hline & $\mathrm{DAN}+\mathrm{CST}$ & 3.0 & - & $>24$ & $>24$ & $>24$ & - & - & - \\
\hline \multirow[t]{3}{*}{ E245 } & DAN & 0.5 & 1.5 & 2.5 & 10.2 & 14.1 & 0.6 & 5.2 & 8.9 \\
\hline & $\mathrm{CST}$ & 3.5 & 2.4 & -0.1 & $>24$ & $>24$ & 0.9 & $>24$ & $>24$ \\
\hline & $\mathrm{DAN}+\mathrm{CST}$ & 1.5 & - & $>24$ & $>24$ & $>24$ & - & - & - \\
\hline \multirow[t]{3}{*}{$E 246$} & DAN & 1.7 & 2.5 & 7 & 9.9 & 10.6 & 8.1 & 8.8 & $>24$ \\
\hline & CST & 3.2 & $>24$ & 10.7 & $>24$ & $>24$ & $>24$ & $>24$ & $>24$ \\
\hline & $\mathrm{DAN}+\mathrm{CST}$ & 2.9 & - & $>24$ & $>24$ & $>24$ & - & - & - \\
\hline \multirow[t]{3}{*}{ E269 } & DAN & 1.3 & 2.3 & 4.3 & 8.6 & 11.7 & 4.6 & 9.7 & 8.2 \\
\hline & CST & 2.5 & 6.1 & 4.9 & 4.7 & 4.6 & 0.4 & 0 & -0.3 \\
\hline & $\mathrm{DAN}+\mathrm{CST}$ & 1.6 & - & $>24$ & $>24$ & $>24$ & - & - & - \\
\hline \multirow[t]{3}{*}{ E306 } & DAN & 0.8 & 1.1 & 2.2 & 3.2 & 3.2 & 1.2 & 3.9 & 4 \\
\hline & $\mathrm{CST}$ & 2.6 & 4.0 & $>24$ & 0.9 & $>24$ & $>24$ & $>24$ & $>24$ \\
\hline & $\mathrm{DAN}+\mathrm{CST}$ & 4.7 & - & $>24$ & $>24$ & $>24$ & - & - & - \\
\hline
\end{tabular}

and $66 \%$, respectively.

The MPC values are shown in table 2. The MPCs of DAN alone ranged from $0.512 \mu \mathrm{g} / \mathrm{ml}$ to $32 \mu \mathrm{g} / \mathrm{ml}$, and from $0.016 \mu \mathrm{g} / \mathrm{ml}$ to $4 \mu \mathrm{g} / \mathrm{ml}$ in combination. The MPCs of CST alone ranged from $32 \mu \mathrm{g} / \mathrm{ml}$ to $256 \mu \mathrm{g} / \mathrm{ml}$, and from $16 \mu \mathrm{g} /$ $\mathrm{ml}$ to $64 \mu \mathrm{g} / \mathrm{ml}$ in combination. DAN+CST reduced MPCs of DAN by 4- to 32- fold and MPCs of CST by 2- to 4- fold. MSW concentrations of DAN+CST were up to 1000 -fold lower than those for DAN alone (table 2). The combination reduced MSW concentrations of CST up to 8-fold. DAN+CST increased MPIs of DAN and CST by 2- to 8- 
fold, except two E.coli isolates (E175, E245) with lower MPIs of DAN+CST than those CST alone.

The PAE and PA-SME results for each antimicrobial alone and in combination for each strain are shown in table 3. The mean PAE values for DAN and CST alone at 1xMICs were $1.3 \mathrm{~h}$ and $2.2 \mathrm{~h}$, respectively. The mean PAE value of DAN+CST against E.coli isolates was 2.7 and slightly higher than the antimicrobials alone. There is no significant difference between PAEs of the antimicrobials alone at $1 \mathrm{xMIC}$ and $4 \mathrm{xMIC}$. PA-SMEs of CST at $0.3 \mathrm{xMIC}$ were significantly longer than its PAE at $1 x M I C(p<0.028)$. PASMEs of DAN+CST at all sub-MIC concentration were significantly longer than the PAEs of DAN+CST at 1xMIC (p $<0.001)$, DAN at both 1xMIC and 4xMIC (p < 0.001, p < $0.001)$, and CST at 1xMIC $(\mathrm{p}<0.001)$.

\section{Discussion}

Effective treatment of infectious diseases is difficult in case of emergence of antimicrobial resistance in bacterial population and as a consequence of this situation the morbidity and mortality increases. The incidence of infections caused by MDR Gram-negative bacteria has increased worldwide over the last decade. ${ }^{18}$ For instance, E.coli and K. pneumoniae isolates were frequently resistant to at least one of the antimicrobials tested or had combined resistance against main antimicrobial groups (third-generation cephalosporins, FQs and aminoglycosides). ${ }^{7}$ Therefore, restoring the efficacy of available antimicrobials against Gram-negative bacteria has become increasingly important.

By checkerboard method, DAN+CST combination exhibited synergistic activity against five of the six E.coli isolates with 0.15-0.50 FICI. As a similar combination, CIP+CST was effective in treatment of infections caused by Gram-negative bacilli and Staphylococcus aureus. ${ }^{19}$ D'Souza et al. ${ }^{5}$ also reported that CIP+CST showed mostly synergistic effect against P. aeruginosa in the checkerboard tests. Wei et al. observed that LVX+CST showed synergistic activity against bla ${ }_{\text {OXA-23 }}$ and bla ${ }_{\text {OXA-51 }}$ positive Acinetobacter baumannii clinical isolates with 0.37 FICI. ${ }^{11}$ After $6 \mathrm{~h}$ of exposure in time-kill assays, DAN+CST had synergistic activity against five of six E.coli isolates as observed in checkerboard tests. In contrast to this, antagonism was detected at $24 \mathrm{~h}$ for five of the six E.coli isolates by the timekill method. MICs were reduced 16-64-fold for DAN and 2-fold for CST in the combination, and were below the clinical breakpoint of individual compound. High synergy incidence can be explained by either E.coli isolates used in this study were at susceptibility borderline or rapid permeabilization of the outer cell membrane caused by CST that allows enhanced penetration and activity of DAN. There was no significant correlation between resistance determinants and interactions of antimicrobials in the combination as reported previously by D'Souza et al. ${ }^{5}$ DAN+CST combination was synergistic against only susceptible E.coli isolates while antagonism was observed for gyrA-, parCand oqxB-containing MDR E.coli isolates by time-kill assays.

$\mathrm{MBC}$ enables to determine the inhibitor or killing potential of antimicrobials on bacterial population and provides fundamental data to predict bacteriostatic/bactericide effect of antimicrobials. ${ }^{20} \mathrm{MBC}$ test results are mostly similar to time-kill assay results. In contrast to this, bactericide-synergistic effect of DAN+CST at $6 \mathrm{~h}$ of time-kill assays was greater than the same effect in the MBC tests. Antimicrobial combinations reduce the MBCs of individual compound and thus effective therapy can be provided with lower concentrations of antimicrobials. ${ }^{21}$ In the present study, MBCs of DAN in the combination was up to 128 -fold lower than DAN alone. Antimicrobial activity was defined as bactericidal and bacteriostatic for $\mathrm{MBC} / \mathrm{MIC}$ ratios $1-4$ and $\geq 8$, respectively. ${ }^{22}$ In the present study, DAN alone exerted bactericidal activity against all E.coli isolates while CST alone and DAN+CST showed bactericidal activity against 4 of six E.coli isolates.

The MPC was defined as the lowest antimicrobial concentration that prevented the visible growth of mutant colonies and a measure of antibiotic potency. ${ }^{23} \mathrm{MPC}$ were expected to be below the clinical breakpoints for an effective antimicrobial treatment. ${ }^{24}$ MPCs of DAN in the combination were below the clinical breakpoints for four of the six E.coli isolates. However, MPCs of CST in the combination were not below the clinical breakpoints for any of E.coli isolates in this study. MPIs of the DAN+CST ranged from 8 to 64 for the E.coli isolates. Zhanel et al. ${ }^{25}$ reported that the MPI for LVX alone was 4 to 8 for P. aeruginosa; when a CST was used in combination with LVX, the MPI of the combination treatment showed a 4- to 8-fold decrease. Cai et al. ${ }^{26}$ showed that the MPI of CST tested alone was 64 or $>64$ for CST-susceptible MDR A. baumannii, and following the addition of LVX at relatively low concentrations, the MPI showed a 4- to 8-fold decrease, and CST-resistant A. baumannii were not selected. In contrast to this, DAN+CST did not decrease the MPIs of the antimicrobials alone and even an increase of MPI was detected for one isolate (E.coli E222). In the present study, MPC of DAN in the combination was $0.016 \mu \mathrm{g} / \mathrm{ml}$ for the susceptible E.coli isolate and ranged from 1 to $4 \mu \mathrm{g} / \mathrm{ml}$ for gyrA mutation containing- $E$. coli isolates. The highest MPI of DAN+CST was $64 \mu \mathrm{g} / \mathrm{ml}$ 
and detected for double mutations-containing E.coli isolate in gyrA gene. Resistance determinants such as gyrA mutations and qnr genes can increase MPCs of antimicrobials up to 8-fold depending on the presence of resistance determinant alone or together. ${ }^{27}$

The PAE is dependent on the concentration of antimicrobial, the exposure time, the bacterial species/strain and the antimicrobial used for PAE test. Aminoglycosides, FQs and protein-synthesis inhibitors have longer PAE times. ${ }^{13}$ In this study, the mean PAE of DAN (1.3 h) against resistant E.coli isolates was found slightly higher than PAEs of FQs (0.29-0.32 h) reported by Harada et al..$^{28}$ The mean PAE of CST $(2.2 \mathrm{~h})$ at 1xMIC was shorter than $3.9 \mathrm{~h}$ reported previously for multidrug-resistant A. baumannii. ${ }^{29}$ There was no statistically difference between PAEs at 1xMIC and $4 x M I C$ in the present study. The mean PAE value of DAN plus CST against E.coli isolates was 2.7 and slightly higher than the antimicrobials alone. However, this increase was not found statistically meaningful. As reported previously, the use of antibiotics in combination with CST at MICs increased the duration of PAE when tested alone, this increase has not been statistically meaningful. ${ }^{30}$ In the other hand, the results of this study showed that PAEs of the combination at sub-MICs were significantly longer than PAEs of the antimicrobials alone.

\section{Conclusions}

In conclusion, DAN+CST combination was found synergistic against MDR E.coli in FIC tests and in the first sampling time of time-kill assays. In addition, the combination exerted bactericidal activity on all MDR E.coli isolates tested. Lower MPC and narrow MSW values showed that the emergence of resistant sub-populations was significantly reduced by $\mathrm{DAN}+\mathrm{CST}$ combination. The other beneficial contribution of the combination was that PA-SME times were significantly prolonged at all sub-MIC concentrations tested. The data provided from this study showed that $\mathrm{DAN}+\mathrm{CST}$ can be considered as a reserve drug combination against MDR E.coli isolates causing various important infectious diseases in veterinary medicine. However, in vivo studies needed to be performed for establishing a better correlation between in vitro studies and clinical effects of the combination.

\section{Funding}

This work was supported by the Scientific and Technological Research Council of Turkey (TUBITTAK) (TOVAG110O478, BIDEB-2211).

\section{References}

1. Sahinturk P, Arslan E, Buyukcangaz E, et al. High level fluoroquinolone resistance in Escherichia coli isolated from animals in Turkey is due to multiple mechanisms. Turk J Vet Anim Sci. 2016;40:214-218.

2. Elemam A, Rahimian J, Doymaz MJ. In vitro evaluation of antibiotic synergy for polymyxin B-resistant carbapenemase-producing Klebsiella pneumoniae. Clin Microbiol. 2010;48(10):3558-3562.

3. Betts JW, Phee LM, Hornsey M, Woodford N, Wareham DW. In vitro and in vivo activities of tigecycline-colistin combination therapies against carbapenem-resistant Enterobacteriaceae. Antimicrob Agents Chemother. 2014;58(6):3541-3546.

4. Lee H, Roh KH, Hong SG, et al. In vitro synergistic effects of antimicrobial combinations on extensively drug-resistant Pseudomonas aeruginosa and Acinetobacter baumannii isolates. Ann Lab Med. 2016;36:138144.

5. D'Souza BB, Padmaraj SR, Rekha PD, Tellis RC, Prabhu S, Pothen P. In vitro synergistic activity of colistin and ceftazidime or ciprofloxacin against multidrug-resistant clinical strains of Pseudomonas aeruginosa. Microb Drug Resist. 2014;20(6):550-554.

6. European Medicines Agency. Updated advice on the use of colistin products in animals 3 within the European Union: development of resistance 4 and possible impact on human and animal health. EMA 2016;231573.

7. Cassir N, Rolain JM, Broqui P. A new strategy to fight antimicrobial resistance: the revival of old antibiotics. Front Microbiol. 2014;5:551.

8. Kempf I, Jouy E, Chauvin C. Colistin use and colistin resistance in bacteria from animals. Int J Antimicrob Agents. 2016;48:598-606.

9. Cengiz M, Sahinturk P, Sonal S, Buyukcangaz E, Sen A, Arslan E. In vitro bactericidal activity of enrofloxacin against gyrA mutant and qnr-containing Escherichia coli isolates from animals. Vet Rec. 2013;172(18):474.

10. Dosler S, Karaaslan E, Gerceker AA. Antibacterial and anti-biofilm activities of melittin and colistin, alone and in combination with antibiotics against Gram-negative bacteria. J Chemother. 2016;28(2):95103.

11. Wei W, Yang H, Hu L, Ye Y, Li J. Activity of levofloxacin in combination with colistin against Acinetobacter baumannii: In vitro and in a Galleria mellonella model. J Microbiol Immunol Infect. 2015;15:1684-1692.

12. Dalhoff A, Schmitz FJ. In vitro antibacterial activity and pharmacodynamics. Eur J Clin Microbiol. 2003;22(4):203-221. 
13. Odenholt I. Pharmacodynamic effects of subinhibitory antibiotic concentrations. Int J Antimicrob Agents 2001;17:1-8.

14. Bambeke FV, Pages JM, Lee VJ. Inhibitors of bacterial efflux pumps as adjuvants in antibiotic treatments and diagnostic tools for detection of resistance by efflux. Recent Pat Antiinfect Drug Discov. 2006;1:157-175.

15. Cengiz M, Sahinturk P. Assessment of synergistic interactions of danofloxacin and orbifloxacin against quinolone-resistant Escherichia coli isolated from animals by the checkerboard and time-kill methods. J Antibiot. 2013;66:629-631.

16. Blondeau JM, Zhao X, Hansen G, Drlica K. Mutant prevention concentrations of fluoroquinolones for clinical isolates of Streptococcus pneumoniae. Antimicrob Agents Chemother. 2001;45(2):433-438.

17. Lorian V, ed. In vitro determination of PAE. In: Antibiotics in laboratory medicine 4th ed. Maryland: 1996.

18. Doyle ME. Multidrug-resistant pathogens in the food supply. Foodborne Pathog Dis. 2015; 12:261-279.

19. Prentice HG, Hann IM, Nazareth B, Paterson P, Bhamra A, Kibbler CC. Oral ciprofloxacin plus colistin: prophylaxis against bacterial infection in neutropenic patients. A strategy for the prevention of emergence of antimicrobial resistance. Br J Haematol. 2001;115(1):46-52.

20. Levison ME, Levison JH. Pharmacokinetics and pharmacodynamics of antibacterial agents. Infect Dis Clin North Am. 2009;23(4):791.

21. Ganiere JP, Denuault L. Synergistic interactions between cefalexin and kanamycin in Mueller-Hinton broth medium and in milk. J Appl Microbiol. 2009;107:117-125.

22. Maaland MG, Mo SS, Schwarz S, Guardabassi L. In vitro assessment of chloramphenicol and florfenicol as second-line antimicrobial agents in dogs. J Vet Pharmacol Ther. 2015;38:443-450.

23. Li Y, Zhang Y, Ding $H$, et al. In vitro susceptibility of four antimicrobials against Riemerella anatipestifer isolates: a comparison of minimum inhibitory concentrations and mutant prevention concentrations for ceftiofur, cefquinome, florfenicol, and tilmicosin. BMC Vet Res. 2016;12:250.

24. Credito K, Kosowska-Shick K, McGhee P, Pankush GA, Appelbaum PC. Comparative study of the mutant prevention concentrations of moxifloxacin, levofloxacin, and gemifloxacin against Pneumococci. Antimicrob Agents Chemother. 2010; 54 (2):673-677.

25. Zhanel GG, Mayer M, Laing N, Adam HJ. Mutant prevention concentrations of levofloxacin alone and in combination with azithromycin, ceftazidime, colistin (polymyxin E), meropenem, piperacillin-tazobactam, and tobramycin against Pseudomonas aeruginosa. Antimicrob Agents Chemother. 2006;50(6):2228-2230.

26. Cai Y, Yang J, Kan Q, et al. Mutant prevention concentration of colistin alone and in combination with levofloxacin or tobramycin against multidrug-resistant Acinetobacter baumannii. Int J Antimicrob Agents. 2012;40(5):477-478.

27. Brailes A, Rodríguez-Martínez JM, Velasco C, et al. In Vitro Effect of qnrA1, qnrB1, and qnrS1 Genes on Fluoroquinolone Activity against Isogenic Escherichia coli Isolates with Mutations in gyrA and parC. Antimicrob Agents Chemother. 2010;55:1266-1269.

28. Harada K, Shimizu T, Kataoka Y, Takahashi T. Post-antibiotic effect of orbifloxacin against Escherichia coli and Pseudomonas aeruginosa isolates from dogs. Acta Vet Scand. 2012;54:16.

29. Plachouras D, Giamarellos-Bourboulis EJ, Kentepozidis N, Baziaka F, Vassiliki K, Giamarellou H. In vitro postantibiotic effect of colistin on multidrug-resistant Acinetobacter baumannii. Diagn Microbiol Infect Dis. 2007;57:419-422.

30. Guzel CB, Gerceker AA. Post-antibiotic effect of colistin, alone and in combination with amikacin, on Pseudomonas aeruginosa strains isolated from cystic fibrosis patients. J Antibiot. 2012;65:83-86. 